\title{
Validity of Semiclassical Gravity in the Stochastic Gravity Approach
}

\author{
E. Verdaguer \\ Departament de Física Fonamental and CER en Astrofísica, Física de Partícules i Cosmologia, \\ Universitat de Barcelona, Av. Diagonal 647, 08028 Barcelona, Spain
}

Received on 20 December, 2004

\begin{abstract}
In semiclassical gravity the back-reaction of the classical gravitational field interacting with quantum matter fields is described by the semiclassical Einstein equations. A criterion for the validity of semiclassical gravity based on the stability of the solutions of the semiclassical Einstein equations with respect to quantum metric perturbations is discussed. The two-point quantum correlation functions for the metric perturbations can be described by the Einstein-Langevin equation obtained in the framework of stochastic gravity. These correlation functions agree, to leading order in the large $N$ limit, with the quantum correlation functions of the theory of gravity interacting with $N$ matter fields. The Einstein-Langevin equations exhibit runaway solutions and methods to deal with these solutions are discussed. The validity criterion is used to show that flat spacetime as a solution of semiclassical gravity is stable and, consequently, a description based on semiclassical gravity is a valid approximation in that case.
\end{abstract}

\section{INTRODUCTION}

Semiclassical gravity describes the interaction of the gravitational field as a classical field with quantum matter fields. For a free quantum field this theory is robust in the sense that it is consistent and fairly well understood $[1,2]$. The gravitational field is described by the semiclassical Einstein equation which has as a source the expectation value in some quantum state of the matter stress tensor operator. The semiclassical theory is in some sense unique as a theory where the gravitational field is classical. In fact, a classical gravitational field interacts with other fields through their stress tensors, and the only reasonable c-number stress tensor that one may construct [3-5] with the stress tensor operator of a quantum field is its expectation value in some quantum state. However, the scope and limits of the theory are not so well understood because we still lack a fully well understood quantum theory of gravity. It is assumed that the semiclassical theory should break down at Planck scales, which is when simple order of magnitude estimates suggest that the quantum effects of gravity cannot be ignored: the gravitational energy of a quantum fluctuation of energy in a Planck size region, determined by the Heisenberg uncertainty principle, is of the same order of magnitude as the energy of the fluctuation itself.

From the semiclassical Einstein equations it seems also clear that the semiclassical theory should break down when the quantum fluctuations of the stress tensor are large. Ford [6] was among the first to have emphasized the importance of these quantum fluctuations. It is less clear, however, how to quantify the size of these fluctuations. Thus, Kuo and Ford [7] used the variance of the fluctuations of the stress tensor operator compared to the mean value as a measure of the validity of semiclassical gravity. As pointed out by $\mathrm{Hu}$ and Phillips $[8,9]$ such a criterion should be refined by considering the back reaction of those fluctuations on the metric. Ford and collaborators also noticed that the metric fluctuations associated to the matter fluctuations can be meaningfully classified as "active" [10-12] and "passive" [6, 7, 13-15].

A different approach to the validity of semiclassical gravity was taken by Horowitz [16, 17] who studied the stability of a semiclassical solution with respect to linear metric perturbations. In the case of a free quantum matter field in its Minkowski vacuum state, flat spacetime is a solution of semiclassical gravity. The equations describing those metric perturbations involve higher order derivatives, and Horowitz found unstable "runaway" solutions that grow exponentially with characteristic timescales comparable to the Planck time; see also the analysis by Jordan [18]. Later, Simon [19, 20], argued that those unstable solutions lie beyond the expected domain of validity of the theory and emphasized that only those solutions which resulted from truncating perturbative expansions in terms of the square of the Planck length are physically acceptable $[19,20]$. Further discussion was provided by Flanagan and Wald [21], who advocated the use of an "order reduction" prescription first introduced by Parker and Simon [22]. More recently Anderson, Molina-París and Mottola have taken up the issue of the validity of semiclassical gravity [23] again. Their starting point is the fact that the semiclassical Einstein equation will fail to provide a valid description of the dynamics of the mean spacetime geometry whenever the higher order radiative corrections to the effective action, involving loops of gravitons or internal graviton propagators, become important. Next, they argue qualitatively that such higher order radiative corrections cannot be neglected if the metric fluctuations grow without bound. Finally, they propose a criterion to characterize the growth of the metric fluctuations, and hence the validity of semiclassical gravity, based on the stability of the solutions of the linearized semiclassical equation. Following these approaches the Minkowski metric is shown to be a stable solution of semiclassical gravity with respect to small metric perturbations.

As emphasized in Ref. [23] the above criteria may be understood as criteria within semiclassical gravity itself. It is certainly true that stability is a necessary condition for the validity of a semiclassical solution, but one may also look for criteria within extensions of semiclassical gravity. In the absence of a quantum theory of gravity such criteria may be found in more modest extensions. Thus, Ford [6] considered graviton production in linearized quantum gravity and compared the results with the production of gravitational waves in semiclassical gravity. Ashtekar [24] and Beetle [25] found large quantum gravity effects in three-dimensional quantum 
gravity models. In a recent paper [26] (see also Ref. [27]) we advocate for a criteria within the stochastic gravity approach. Stochastic semiclassical gravity extends semiclassical gravity by incorporating the quantum stress tensor fluctuations of the matter fields; see Refs. [28, 29] for reviews.

It turns out that this validity criteria is equivalent to the validity criteria that one might advocate within the large $N$ expansion, that is the theory describing the interaction of the gravitational field with $N$ identical matter fields. In the leading order, namely the limit in which $N$ goes to infinity and the gravitational constant is appropriately rescaled, the theory reproduces semiclassical gravity. Thus, a natural extension of semiclassical gravity is provided by the next to leading order. It turns out that the symmetrized two-point quantum correlations of the metric perturbations in the large $N$ expansion are equivalent to the two-point stochastic metric fluctuations predicted by stochastic gravity. Our validity criterion can then be summarized as follows: a solution of semiclassical gravity is valid when it is stable with respect to quantum metric perturbations. This criterion implies to consider the quantum correlation functions of the metric perturbations.

It is important to emphasize that the above validity criterion incorporates in a unified and self-consistent way the two main ingredients of the criteria exposed above. Namely, the criteria based on the quantum stress tensor fluctuations of the matter fields, and the criteria based on the stability of semiclassical solutions against classical metric perturbations. In the following discussion we will argue that the former is incorporated through the so called induced fluctuations and the later though the so called intrinsic fluctuations. These correspond to Ford's "passive" and "active" fluctuations, respectively. We will see that symmetrized quantum two-point metric fluctuations can always be decomposed as a sum of induced and intrinsic fluctuations.

The paper is organized as follows. In section II we briefly review the main ingredients of semiclassical gravity. In section III we introduce stochastic gravity as a theory that goes beyond semiclassical theory by incorporating the fluctuations of the quantum stress tensor operator. In section IV our validity criterion is applied to the study of flat spacetime as a solution of semiclassical gravity. The problem of the runaway solutions and methods to deal them is discussed. Throughout the paper in order to emphasize the qualitative aspects we use a simplified notation without tensorial indices and for a few points we also use qualitative arguments and order of magnitude estimates. We refer the reader to the papers [26-29] were the technical details, as well as many subtleties that cannot be summarized here, are provided. Our metric and curvature conventions are those of Ref. [30], and we use $\hbar=c=1$.

\section{SEMICLASSICAL GRAVITY}

At present semiclassical gravity cannot be rigorously derived, but, it can be formally justified in several ways. One of them is the leading order in the large $N$ expansion [31], where $N$ is the number of independent free quantum fields which interact with gravity only. In this limit, after path integration one arrives at a theory in which formally the gravitational field can be treated as a c-number and the quantum fields are fully quantized.

Semiclassical gravity can be summarized as follows. Let $g$ be the metric tensor and $\hat{\phi}$ a scalar field operator. The semiclassical Einstein equation as the dynamical equation that describes the back-reaction of quantum matter on the metric $g$ can be written as

$$
G_{g}=\kappa\left\langle\hat{T}^{R}\right\rangle_{g}
$$

where $\hat{T}=T\left[\hat{\phi}^{2}\right]$ is the matter stress tensor in a simplified notation, which is quadratic in the field operator $\hat{\phi}$, and $\kappa=8 \pi G$, where $G$ is Newton's constant. This operator, being the product of distribution valued operators, is ill defined and needs to be regularized and renormalized, the $R$ in $\hat{T}^{R}$ means that the operator has been renormalized. The angle brackets on the right hand side mean that the expectation value of the stress tensor operator is computed in some quantum state, say $|\psi\rangle$, compatible with the geometry described by the metric $g$. On the left hand side $G_{g}$ stands for the Einstein tensor of the metric $g$ together with the cosmological constant term and other terms quadratic in the curvature which are generally needed to renormalize the matter stress tensor operator. The quantum field operator $\hat{\phi}$ propagates in the background defined by the metric $g$, it thus satisfies a Klein-Gordon equation,

$$
\left(\square_{g}-m^{2}\right) \hat{\phi}=0,
$$

where $\square_{g}$ stands for the D'Alambert operator in the background of $g$ and $m$ is the mass of the scalar field. A solution of semiclassical gravity consists of the set $(g, \hat{\phi},|\psi\rangle)$ where $g$ is a solution of Eq. (1), $\hat{\phi}$ is a solution of Eq. (2) and $|\psi\rangle$ is the quantum state in which the expectation value of the stress tensor in Eq. (1) is computed.

As we recalled in the introduction this theory is in some sense unique as a theory that describes the interaction of a classical gravitational field with quantum matter. As an effective theory it should break down at Planck scales. Also, from the right hand side of the semiclassical Einstein equation it seems clear that the theory should also break down when the fluctuations of the quantum stress tensor are large. This has been emphasized by Ford and collaborators, and may be illustrated by the example of Ref. [6] as follows.

Let us assume a quantum state formed by an isolated system which consists of a superposition with equal amplitude of one configuration with mass $M_{1}$ and another with mass $M_{2}$. Semiclassical theory as described in Eq. (1) predicts that the gravitational field of this system is produced by the average mass $\left(M_{1}+M_{2}\right) / 2$, that is a test particle will move on the background spacetime produced by such a source. However one would expect that if we send a succession of test particles to probe the gravitational field of the above system half of the time they would react to the field of a mass $M_{1}$ and the other half to the field of a mass $M_{2}$. If the two masses differ substantially the two predictions are clearly different, note that the fluctuations in mass of the quantum state is of the order of $\left(M_{1}-M_{2}\right)^{2}$. Although the previous example is suggestive a word of caution should be said in order not to take it too 
literary. In fact, if the previous masses are macroscopic the quantum system decoheres very quickly [32] and instead of a pure quantum state it is described by a density matrix which diagonalizes in a certain pointer basis. Thus for observables associated to this pointer basis the matrix density description is equivalent to that provided by a statistical ensemble. In any case, however, from the point of view of the test particles the predictions differ from that of the semiclassical theory.

\section{STOCHASTIC GRAVITY}

The purpose of stochastic (semiclassical) gravity is to be able to deal with the situation of the previous example when the predictions of the semiclassical theory may be inaccurate. Consequently, our first point is to characterize the quantum fluctuations of the stress tensor.

The physical observable that measures these fluctuations is $\left\langle\hat{T}^{2}\right\rangle-\langle\hat{T}\rangle^{2}$. To make this more precise let us introduce the tensor operator $\hat{t} \equiv \hat{T}-\langle\hat{T}\rangle \hat{I}$, where $\hat{I}$ is the identity operator, then we introduce the noise kernel as the four-index bi-tensor defined as the expectation value of the anticommutator of the operator $\hat{t}$ :

$$
N(x, y)=\frac{1}{2}\langle\{\hat{t}(x), \hat{t}(y)\}\rangle_{g} .
$$

Thus, the noise kernel is the symmetrized connected part of the two-point quantum correlation function of the stress tensor operator with respect to the state of the matter fields. The subindex $g$ here means that this expectation value in taken in a background metric $g$. An important property of the symmetric bi-tensor $N(x, y)$ is that it is finite because the tensor operator $\hat{t}$ is finite since the ultraviolet divergences of $\hat{T}$ are cancelled by the substraction of $\langle\hat{T}\rangle$. Since the operator $\hat{T}$ is selfadjoint $N(x, y)$, which is the expectation value of an anticommutator, is real and positive semi-definite [28]. Thus, when considering the inverse kernel $N^{-1}(x, y)$, one must work in the subspace obtained from the eigenvectors which have strictly positive eigenvalues when the noise kernel is diagonalized. The last property allows for the introduction of a classical Gaussian stochastic tensor $\xi$ defined by

$$
\langle\xi(x)\rangle_{s}=0, \quad\langle\xi(x) \xi(y)\rangle_{s}=N(x, y) .
$$

This stochastic tensor is symmetric and divergenceless, $\nabla \cdot \xi=$ 0 , as a consequence of the fact that the stress tensor operator is divergenceless. The subindex $s$ means that the expectation value is just a classical stochastic average. Note that we assume that $\xi$ is Gaussian just for simplicity in order to include the main effect of the quantum fluctuations.

The idea now is simple we want to modify the semiclassical Einstein equation (1) by introducing a linear correction to the metric tensor $g$, such as $g+h$, which accounts consistently for the fluctuations of the stress tensor. The simplest equation is,

$$
G_{g+h}=\kappa\left(\left\langle\hat{T}^{R}\right\rangle_{g+h}+\xi\right),
$$

where $g$ is assumed to be a solution of equation (1). This stochastic equation must be thought of as a linear equation for the metric perturbation $h$ which will behave, consequently, as a stochastic field tensor. Note that the tensor $\xi$ is not a dynamical source, since it has been defined in the background metric $g$ which is a solution of the semiclassical equation. Note also that this source is divergenceless with respect to the metric, and it is thus consistent to write it on the right hand side of the Einstein equation. This equation is gauge invariant with respect to diffeomorphisms defined by any field on the background spacetime [33]. If we take the statistical average of equation (5) it becomes just the semiclassical equation for the perturbed metric $g+h$ where now the expectation value of $\hat{T}$ is taken in the perturbed spacetime.

The stochastic equation (5) is known as the EinsteinLangevin equation. To linear order in $h$ we have [33],

$$
\left\langle\hat{T}^{R}\right\rangle_{g+h}(x)=-2 \int H\left(x, x^{\prime}\right) \cdot h\left(x^{\prime}\right),
$$

where the kernel $H\left(x, x^{\prime}\right)$ has three terms, one of them is proportional to the imaginary part of the expectation value of the time ordered two-point stress tensor, $\operatorname{Im}\left\langle T\left(\hat{T}(x) \hat{T}\left(x^{\prime}\right)\right)\right\rangle$, the second term is proportional to the expectation value of the stress tensor commutator, $\left\langle\left[\hat{T}(x), \hat{T}\left(x^{\prime}\right)\right]\right\rangle$, and the third is proportional to the functional derivative of $\langle\hat{T}\rangle$ with respect to the metric (excluding the implicit dependence on the metric of the field $\hat{\phi}$ ). Of course, this kernel is also the main ingredient of the linearized semiclassical Einstein equation around a given background metric $g$. The other key ingredient in the Einstein-Langevin equation is the noise kernel $N(x, y)$ which defines the stochastic inhomogeneous source of the equation. This kernel should be thought of as a distribution function, the limit of coincidence points has meaning only in the sense of distributions. Explicit expressions of this kernel in terms of the two point Wightman functions are given in Ref. [33] on a general background. Detailed expressions for this kernel in the Minkowski background are given in Ref. [34], and expression based on point-splitting methods have also been given in Refs. $[9,35]$ in other backgrounds.

The Einstein-Langevin equation has been previously derived making use of a formal analogy with open quantum systems and employing the influence functional formalism $[36,37]$. The basis for this approach is a functional formalism known as closed time path, first introduced by Schwinger [38-40], which is an effective action method suitable to derive dynamical equations for expectation values of quantum operators; rather than transition elements as in the standard effective action method. The closed time path formalism was later applied to the problem of back-reaction of quantum fields on the spacetime metric [41-43], in order to derive semiclassical Einstein equations. The formalism was then applied along the lines of the influence functional formalism to derive Einstein-Langevin equations in several contexts [33, 4449]. In Ref. [50] the Einstein-Langevin equation was derived by an axiomatic approach by arguing that it is the only consistent generalization of the semiclassical Einstein equation which takes into account the back-reaction of the matter stress tensor fluctuations to lowest order. We have summarized the axiomatic approach in this section.

The solution of the Einstein-Langevin equation (5), taking 
into account Eq. (6), may be expressed as,

$$
h(x)=h^{0}(x)+\kappa \int G_{R}\left(x, x^{\prime}\right) \cdot \xi\left(x^{\prime}\right),
$$

where $h^{0}$ is a solution of the homogeneous part of equation (5) which contains all the information on the initial conditions, and $G_{R}\left(x, x^{\prime}\right)$ is the retarded propagator with vanishing initial conditions associated with the equation (5). The two-point correlation function for the metric perturbation which is the physically most relevant observable can then be written as:

$$
\begin{aligned}
\langle h(x) h(y)\rangle_{s} & =\left\langle h^{0}(x) h^{0}(y)\right\rangle_{s}+ \\
& \kappa^{2} \int G_{R}\left(x, x^{\prime}\right) \cdot N\left(x^{\prime}, y^{\prime}\right) \cdot G_{R}\left(y, y^{\prime}\right),
\end{aligned}
$$

where the first average, $\left\langle h^{0}(x) h^{0}(y)\right\rangle_{s}$, is taken with respect to the initial conditions.

It turns out that going to leading order in $1 / N$, in the large $N$ expansion, one can show that the stochastic correlation functions for the metric perturbations obtained from the Einstein-Langevin equation coincide with the symmetrized two-point quantum correlation functions of the metric perturbations. The details of the derivation will be given in Ref. [51] and are summarized in Ref. [26] for the particular case of a Minkowski background, to which we will restrict in section IV. In this case $\kappa$ in Eq. (7) has to be replaced by the rescaled gravitational coupling constant $\bar{\kappa}=N \kappa$ and the noise kernel for a single field $N(x, y)$ must be replaced by $(1 / N) N(x, y)$. Thus, we have that the symmetrized two-point quantum correlation function for the metric perturbation is

$$
\frac{1}{2}\langle\{\hat{h}(x), \hat{h}(y)\}\rangle=\langle h(x) h(y)\rangle_{s} .
$$

where the Lorentz gauge condition $\nabla \cdot(h-(1 / 2) \eta \operatorname{Tr} h)=0(\eta$ is the Minkowski metric) as well as some initial condition to fix completely the remaining gauge freedom of the initial state should be implicitly understood.

It should be emphasized that there are two different contributions to the symmetrized quantum correlation function, which are clearly distinguished in Eq. (8). The first contribution is related to the quantum fluctuations of the initial state of the metric perturbations and corresponds to the so called intrinsic fluctuations; here the stochastic average must be taken with respect to the Wigner distribution function that describes the initial quantum state. The second contribution is proportional to the noise kernel, it accounts for the fluctuations of the stress tensor of the matter fields and corresponds to the so called induced fluctuations. These two contributions to the two-point correlation functions is also seen in the description of some quantum Brownian motion models which are typically used as paradigms of open quantum systems [52-54]. Both, the intrinsic and induced fluctuations, play a role in our stability criterion for the solutions of semiclassical gravity.

The full two-point quantum correlation function for the metric $\langle\hat{h}(x) \hat{h}(y)\rangle$ can, in fact, be obtained from the EinsteinLangevin equation. Since this correlation can be given in terms of the antisymmetrized and the symmetrized quantum correlation function we only need the commutator that to leading order in $1 / N$ is independent of the initial state of the metric perturbation and is given by

$$
\frac{1}{2}\langle[\hat{h}(x), \hat{h}(y)]\rangle=i \kappa\left[G_{R}(y, x)-G_{R}(x, y)\right] .
$$

Note that the information on the retarded propagator is already in the linearized semiclassical Einstein equation. That is, Eq. (5) without the stochastic source.

\section{A. A toy model}

To justify Eq. (9) which plays an essential role in our criteria for the validity of semiclassical gravity it is useful to introduce a simple toy model for gravity which minimizes the technical complications. The model is also useful to clarify the role of the noise kernel and illustrate the relationship between the semiclassical, stochastic and quantum descriptions. Let us assume that the gravitational equations are described by a massless scalar field $h$ whose source is another massless scalar field $\phi$ which satisfies the Klein-Gordon equation in flat spacetime $\square \phi=0$. The field stress tensor is quadratic in the field, and independent of $h$. The classical gravitational field equations will be given by

$$
\square h=\kappa T,
$$

where $T$ is now the (scalar) trace of the stress tensor. Note that this is not a self-consistent theory since $\phi$ does not react to the gravitational field $h$. This model obviously differs from the standard linearized theory of gravity discussed previously, where $T$ is also linear in $h$, but it captures some of its key features.

In the Heisenberg representation the quantum scalar field $\hat{h}$ satisfies

$$
\square \hat{h}=\kappa \hat{T} .
$$

Since $\hat{T}$ is quadratic in the field operator $\hat{\phi}$ some regularization procedure has to be assumed in order for Eq. (12) to make sense. Since we work in flat spacetime we may simply use a normal ordering prescription to regularize the operator $\hat{T}$. The solutions of this equation, i.e. the field operator at the point $x$, which we call $\hat{h}_{x}$ in this subsection to avoid confusion with the more standard notation, $\hat{h}(x)$, used in the rest of the paper, may be written in terms of the retarded propagator $G_{x x^{\prime}}$ of the D’Alambertian as,

$$
\hat{h}_{x}=\hat{h}_{x}^{0}+\kappa \int G_{x x^{\prime}} \hat{T}_{x^{\prime}},
$$

where $\hat{h}_{x}^{0}$ is the free field which carries information on the initial conditions and the state of the field. From this solution we may compute, for instance, the symmetric two-point quantum correlation function (the anticommutator)

$$
\left\langle\left\{\hat{h}_{x}, \hat{h}_{y}\right\}\right\rangle=\left\langle\left\{\hat{h}_{x}^{0}, \hat{h}_{y}^{0}\right\}\right\rangle+\kappa^{2} \int G_{x x^{\prime}} G_{y y^{\prime}}\left\langle\left\{\hat{T}_{x^{\prime}}, \hat{T}_{y^{\prime}}\right\}\right\rangle,
$$


where the expectation value is taken with respect to the quantum state in which both fields $\phi$ and $h$ are quantized. We have assumed $\left\langle\hat{h}^{0}\right\rangle=0$ for the free field.

We can now consider the semiclassical theory for this problem. If we assume that $h$ is classical and the matter field is quantum the semiclassical limit may just be described by substituting into the classical equation (11) the stress trace by the expectation value of the scalar stress operator $\langle\hat{T}\rangle$, in some quantum state of the field $\hat{\phi}$. We may simply renormalize the expectation value of $\hat{T}$ using normal ordering, then for the vacuum state of the field $\hat{\phi}$, we would simply have $\langle\hat{T}\rangle_{0}=0$. The semiclassical theory thus reduces to

$$
\square h=\kappa\langle\hat{T}\rangle .
$$

The two point function $h_{x} h_{y}$ that one may derive from this equation depends on the two point function $\left\langle\hat{T}_{x}\right\rangle\left\langle\hat{T}_{y}\right\rangle$ and clearly cannot reproduce the quantum result of Eq. (14) which depends on the expectation value of two-point operator $\left\langle\left\{\hat{T}_{x}, \hat{T}_{y}\right\}\right\rangle$. That is, the semiclassical theory entirely misses the fluctuations of the scalar stress operator $\hat{T}$.

To extend this semiclassical theory in order to account for such fluctuations, we introduce the noise kernel as we did in the previous section. Thus, we define

$$
N_{x y}=\frac{1}{2}\left\langle\left\{\hat{t}_{x}, \hat{t}_{y}\right\}\right\rangle
$$

where $\hat{t} \equiv \hat{T}-\langle\hat{T}\rangle$, and we have used again the sub-index notation to avoid confusion with the noise kernel of the previous section. The bi-scalar $N_{x y}$ is real and positive-semidefinite, as a consequence of $\hat{t}$ being self-adjoint [28]. Consequently we can introduce a Gaussian stochastic field as:

$$
\left\langle\xi_{s}=0, \quad\left\langle\xi_{x} \xi_{y}\right\rangle_{s}=N_{x y} .\right.
$$

where the subscript $s$ means a statistical average.

The extension of the semiclassical equation may be simply performed by adding to the right-hand side of the semiclassical equation (15) the stochastic source $\xi$, which accounts for the fluctuations of $\hat{T}$ as follows,

$$
\square h=\kappa(\langle\hat{T}\rangle+\xi) .
$$

This equation is in the form of a Langevin equation: the field $h$ is classical but stochastic and the observables we may obtain from it are correlation functions for $h$. In fact, the solution of this equation may be written in terms of the retarded propagator as,

$$
h_{x}=h_{x}^{0}+\kappa \int G_{x x^{\prime}}\left(\left\langle\hat{T}_{x^{\prime}}\right\rangle+\xi_{x^{\prime}}\right),
$$

from where the two point correlation function for the classical field $h$, after using the definition of $\xi$ and that $\left\langle h^{0}\right\rangle_{s}=0$, is given by

$$
\left\langle h_{x} h_{y}\right\rangle_{s}=\left\langle h_{x}^{0} h_{y}^{0}\right\rangle_{s}+\frac{\kappa^{2}}{2} \int G_{x x^{\prime}} G_{y y^{\prime}}\left\langle\left\{\hat{T}_{x^{\prime}}, \hat{T}_{y^{\prime}}\right\}\right\rangle .
$$

Note that in writing $\langle\ldots\rangle_{s}$ here we are assuming a double stochastic average, one is related to the stochastic process $\xi$ and the other is related to the free field $h^{0}$ which is assumed also to be stochastic with an initial distribution function to be specified.

Comparing Eqs. (14) and (20) we see that the respective second term on the right-hand side are identical (except for a factor of 2 due to the symmetrization) provided the expectation values are computed in the same quantum state for the field $\hat{\phi}$. The fact that the field $h$ is also quantized in (14) does not change the previous statement; recall that $T$ does not depend on $h$. The nature of the first term on the right-hand sides of equations (14) and (20) is different: in the first case it is the two-point quantum expectation value of the free quantum field $\hat{h}^{0}$ whereas in the second case it is the stochastic average of the two point classical homogeneous field $h^{0}$, which depends on the initial conditions. Now we can still make these terms equal to each other (with the factor of 2) if we assume for the homogeneous field $h^{0}$ a Gaussian distribution of initial conditions such that

$$
\left\langle h_{x}^{0} h_{y}^{0}\right\rangle_{s}=\frac{1}{2}\left\langle\left\{\hat{h}_{x}^{0}, \hat{h}_{y}^{0}\right\}\right\rangle .
$$

This Gaussian stochastic field $h^{0}$ can always be defined due to the semi-positivity of the anti-commutator. Thus, under this assumption on the initial conditions for the field $h$ the two point correlation function of Eq. (20) equals the quantum expectation value of Eq. (14) exactly. Thus, we have

$$
\frac{1}{2}\left\langle\left\{\hat{h}_{x}, \hat{h}_{y}\right\}\right\rangle=\left\langle h_{x} h_{y}\right\rangle_{s},
$$

which may be compared to Eq. (9). Comparing with the linearized theory of gravity described in the previous section we see that $\langle T\rangle$ depends also on $h$, both explicitly and also implicitly through the coupling of $\phi$ with $h$. The retarded propagator here $G_{x x^{\prime}}$ is then replaced by the propagator $G_{R}\left(x, x^{\prime}\right)$ of the previous section and the functions $h^{0}$, which are here the free metric perturbations are replaced by the homogeneous solutions of the previous section.

\section{STABILITY OF FLAT SPACETIME}

Let us now apply our validity criterion to flat spacetime. One particularly simple and interesting solution of semiclassical gravity is the Minkowski metric. In fact, when the quantum fields are in the Minkowski vacuum state one may take the renormalized expectation value of the stress tensor $\left\langle T^{R}\right\rangle=0$ (this is equivalent to assuming that the cosmological constant is zero) and the Minkowski metric $\eta$ is a solution of the semiclassical Einstein equation (1). Thus, we can look for the stability of flat spacetime against quantum matter fields. According to the criteria we have established we have to look for the behavior of the two-point quantum correlations for the metric perturbations $h$ over the Minkowski background which are given by Eqs. (8) and (9). As we have emphasized several times these fluctuations separate in two parts: the first term on the right hand side of Eq. (8) corresponds to the intrinsic fluctuations, and the second term corresponds to the induced fluctuations. 


\section{A. Intrinsic fluctuations}

Let us first consider the intrinsic fluctuations,

$$
\left\langle h^{0}(x) h^{0}(y)\right\rangle_{s},
$$

where $h^{0}$ are the homogeneous solutions of the EinsteinLangevin equation (5), or equivalently the linearly perturbed semiclassical equation, and the statistical average is taken with respect to the Wigner distribution that describes the initial quantum state of the metric perturbations. Since these solutions are described by the linearized semiclassical equation around flat spacetime we can make use of the results derived in Refs. [16, 21, 23]. The solutions for the case of a massless scalar field were first discussed in Ref. [16] and an exhaustive description can be found in Appendix A of Ref. [21]. Decomposing the metric perturbation into scalar, vectorial and tensorial parts and computing the linearized Einstein tensor, one gets a vanishing result for the vectorial part of the metric perturbation; the scalar and tensorial components of the metric perturbation give rise, respectively, to the scalar and tensorial components of the linearized Einstein tensor. The vectorial part is found to vanish whereas the scalar and tensorial contributions for a massless and conformally coupled scalar field (see Ref. [21] for the massless case with arbitrary coupling and Refs. [23, 34] for the general massive case) satisfy the following equations:

$$
\begin{gathered}
\left(1+12 \kappa \bar{\beta} p^{2}\right) \tilde{G}^{(\mathrm{S})}(p)=0, \\
\lim _{\varepsilon \rightarrow 0^{+}}\left(1+\frac{\kappa p^{2}}{960 \pi^{2}} \ln \frac{p^{2}}{\mu^{2}}\right) \tilde{G}^{(\mathrm{T})}(p)=0,
\end{gathered}
$$

where in the last equation the prescription that the time component of $p$ has a small imaginary part, $p^{0}+i \varepsilon$, is taken. Here $\tilde{G}(p)$ stands for the Fourier transform of the linearized Einstein tensor, the upper indices $S$ and $T$ stand for scalar and tensorial respectively, $\bar{\beta}$ is a dimensionless renormalized parameter that multiplies some of the quadratic terms in the curvature in the effective action for the gravitational field, and $\mu$ is a renormalization mass scale. See Ref. [26] for a more complete description.

For the scalar component when $\bar{\beta}=0$ the only solution is $\tilde{G}^{(\mathrm{S})}(p)=0$. When $\bar{\beta}>0$ the solutions for the scalar component exhibit an oscillatory behavior in spacetime coordinates which corresponds to a massive scalar field with $\mathrm{m}^{2}=$ $(12 \kappa|\bar{\beta}|)^{-1}$; for $\bar{\beta}<0$ the solutions correspond to a tachyonic field with $m^{2}=-(12 \kappa|\bar{\beta}|)^{-1}$ : in spacetime coordinates they exhibit an exponential behavior in time, growing or decreasing, for wavelengths larger than $4 \pi(3 \kappa|\bar{\beta}|)^{1 / 2}$, and an oscillatory behavior for wavelengths smaller than $4 \pi(3 \kappa|\bar{\beta}|)^{1 / 2}$. On the other hand, the solution $\tilde{G}^{(\mathrm{S})}(p)=0$ is completely trivial since any scalar metric perturbation $\tilde{h}(p)$ giving rise to a vanishing linearized Einstein tensor can be eliminated by a gauge transformation as explained in Ref. [26].

For the tensorial component, when $\mu \leq \mu_{\text {crit }}=$ $l_{p}^{-1}(120 \pi)^{1 / 2} e^{\gamma}$, where $l_{p}$ is the Planck length $\left(l_{p}^{2} \equiv \kappa / 8 \pi\right)$ the first factor in Eq. (25) vanishes for four complex values of $p^{0}$ of the form $\pm \omega$ and $\pm \omega^{*}$, where $\omega$ is some complex value. We will consider here the case in which $\mu<\mu_{\text {crit }}$; a detailed description of the situation for $\mu \geq \mu_{\text {crit }}$ can be found in Appendix A of Ref. [21]. The two zeros on the upper half of the complex plane correspond to solutions in spacetime coordinates exponentially growing in time, whereas the two on the lower half correspond to solutions exponentially decreasing in time. Strictly speaking, these solutions only exist in spacetime coordinates, since their Fourier transform is not well defined. They are commonly referred to as runaway solutions and for $\mu \sim l_{p}^{-1}$ they grow exponentially in time scales comparable to the Planck time.

In order to deal with those unstable solutions, one possibility is to employ the order reduction prescription [22], which we will briefly summarize in the last subsection. With such a prescription we are left only with the solutions which satisfy $\tilde{G}(p)=0$. The solutions for $\tilde{h}(p)$ simply correspond to free linear gravitational waves propagating in Minkowski spacetime expressed in the transverse and traceless (TT) gauge. When substituting back into Eq. (23) and averaging over the initial conditions we simply get the symmetrized quantum correlation function for free gravitons in the TT gauge for the state given by the Wigner distribution. As far as the intrinsic fluctuations are concerned, it seems that the order reduction prescription is too drastic, at least in the case of Minkowski spacetime, since no effects due to the interaction with the quantum matter fields are left.

A second possibility, proposed by Hawking et al. [55, 56], is to impose boundary conditions which discard the runaway solutions that grow unbounded in time and correspond to a special prescription for the integration contour when Fourier transforming back to spacetime coordinates. Following that procedure we get, for example, that for a massless conformally coupled scalar field with $\bar{\beta}>0$ the intrinsic contribution to the symmetrized quantum correlation function coincides with that of free gravitons plus an extra contribution for the scalar part of the metric perturbations which renders Minkowski spacetime stable but plays a crucial role in providing a graceful exit for inflationary models driven by the vacuum polarization of a large number of conformal fields. Such a massive scalar field would not be in conflict with present observations because, for the range of parameters considered, the mass would be far too large to have observational consequences [55].

\section{B. Induced fluctuations}

The induced fluctuations are described by the second term in Eq. (8). They are induced for the noise kernel that describes the stress tensor fluctuations of the matter fields,

$$
\frac{\bar{\kappa}^{2}}{N} \int G_{R}\left(x, x^{\prime}\right) \cdot N\left(x^{\prime}, y^{\prime}\right) \cdot G_{R}\left(y, y^{\prime}\right),
$$

where we write the expression in the large $N$ limit. The contribution corresponding to the induced quantum fluctuations is equivalent to the stochastic correlation function obtained by considering just the inhomogeneous part of the solution to 
the Einstein-Langevin equation: the second term on the righthand side of Eq. (8). Taking all that into account, it is clear that we can make use of the results for the metric correlations obtained in Ref. [34] by solving the Einstein-Langevin equation. In fact, one should simply take $N=1$ to transform our expressions to those of Ref. [34] and, similarly, multiply the noise kernel in the expressions of that reference by $N$ so that they can be used here, which follows from the fact that we have $N$ independent matter fields.

The same kind of exponential instabilities in the runaway solutions of the homogeneous part of the Einstein-Langevin equation also arise when computing the retarded propagator $G_{\mathrm{R}}$. In order to deal with those instabilities, similar to the case of the intrinsic fluctuations, one possibility is to make use of the order reduction prescription. The Einstein-Langevin equation becomes then $\tilde{G}(p)=\bar{\kappa} \tilde{\xi}(p)$. The second possibility, following the proposal of Hawking et al., is to impose boundary conditions which discard the exponentially growing solutions and translate into a special choice of the integration contour when Fourier transforming back to spacetime coordinates the expression for the propagator. In fact, it turns out that the propagator which results from adopting that prescription coincides with the propagator that was employed in Ref. [34]. Note, however, that this propagator is no longer a strictly retarded propagator since it exhibits causality violations at Planck scales. A more detailed discussion on all these points can be found in Appendix E of Ref. [26].

Following Ref. [34], the Einstein-Langevin equation can be entirely written in terms of the linearized Einstein tensor. The equation involves second derivatives of that tensor, and in terms of its Fourier components $\tilde{G}(p)$ takes the form

$$
(1+F(p)) \cdot \tilde{G}(p)=\bar{\kappa} \tilde{\xi}(p),
$$

where $F$ is a four-index tensor which depends on $p^{2} \ln p^{2}$ when the field is massless and conformally coupled. This reflects the fact that we have second derivatives of the Einstein tensor and the nonlocality of the Einstein-Langevin equation (or also of the perturbed semiclassical equation). From equation (27) one may obtain the correlation functions for $\tilde{G}(p)$, $\langle\tilde{G}(p) \tilde{G}(q)\rangle_{s}$, which are invariant under gauge transformations of the metric perturbations. Writing the linearized Einstein tensor in terms of the metric perturbation, which takes a particularly simple form in the Lorentz gauge, one may derive the correlation functions for $\tilde{h}(p):\langle\tilde{h}(p) \tilde{h}(q)\rangle_{s}$. Finally, the correlation functions in spacetime coordinates can be easily obtained by Fourier transforming these correlations. For massless and conformally coupled matter fields explicit results are given in Ref. [34], they have the general expression:

$$
\langle h(x) h(y)\rangle_{s}=\frac{\bar{\kappa}^{2}}{720 \pi N} \int \frac{e^{i p \cdot(x-y)} P \theta\left(-p^{2}\right)}{\left|1+\left(\bar{\kappa} / 960 \pi^{2}\right) p^{2} \ln \left(p^{2} / \mu^{2}\right)\right|^{2}}
$$

where $P$ is a four-index projection tensor. This correlation function for the metric perturbations is in agreement with the real part of the propagator obtained by Tomboulis in Ref. [57] using a large $N$ expansion.

To estimate this integral let us consider spacelike separated points $x-y=(0, \mathbf{r})$ and introduce the Planck length $l_{p}$. It is not difficult to see [29], that for space separations $|\mathbf{r}| \gg l_{p}$ we have

$$
\langle h(x) h(y)\rangle_{s} \sim \frac{l_{p}^{4}}{|\mathbf{r}|^{4}}
$$

and for $|\mathbf{r}| \sim N l_{p}$ we have

$$
\langle h(x) h(y)\rangle_{s} \sim e^{-|\mathbf{r}| / l_{p}} \frac{l_{p}}{|\mathbf{r}|} .
$$

Since these fluctuations are induced by the matter stress fluctuations we infer that the effect of the matter fields is to suppress metric fluctuations at small scales. On the other hand, at large scales the induced metric fluctuations are small compared to the free graviton propagator which goes like $l_{p}^{2} /|\mathbf{r}|^{2}$.

We thus conclude that, once the instabilities giving rise to the unphysical runaway solutions have been discarded, the fluctuations of the metric perturbations around the Minkowski spacetime induced by the interaction with quantum scalar fields are indeed stable. Instabilities would lead to a divergent result when Fourier transforming back to spacetime coordinates. Note that when the order reduction prescription is used the $p^{2} \ln p^{2}$ terms are absent in the corresponding Eq. (28). Thus, in contrast to the intrinsic fluctuations, there is still a nontrivial contribution to the induced fluctuations due to the quantum matter fields in this case.

\section{Order reduction prescription and large $N$}

Runaway solutions are a typical feature of equations describing back-reaction effects, such is in classical electrodynamics, and are due to higher than two time derivatives in the dynamical equations. In a very schematic way the semiclassical Einstein equations have the form

$$
G_{h}+l_{p}^{2} \ddot{G}_{h}=0
$$

where $G_{h}$ stands for the linearized Einstein tensor over the Minkowski background, say, and we have simplified the equation as much as possible. The second term of the equation is due to the vacuum polarization of matter fields and contains four time derivatives of the metric perturbation. Some specific examples of such an equation are, in momentum space, Eqs. (24) and (25). The order reduction procedure is based on treating perturbatively the terms involving higher order derivatives, differentiating the equation under consideration and substituting back the higher derivative terms in the original equation keeping only terms up to the required order in the perturbative parameter. In the case of the semiclassical Einstein equation, the perturbative parameter is $l_{p}^{2}$. If we differentiate twice Eq. (31) with respect to time it is clear that the second order derivatives of the Einstein tensor are of order $l_{p}^{2}$. Substituting back into the original equation, we get the following equation up to order $l_{p}^{4}: G_{h}=0+O\left(l_{p}^{4}\right)$. Now, there are certainly no runaway solutions but also no effect due to the vacuum polarization of matter fields. Note that the result is not so trivial when there is an inhomogeneous term on 
the right hand side of Eq. (31), this is what happens with the induced fluctuations predicted by the Einstein-Langevin equation.

Semiclassical gravity is expected to provide reliable results as long as the characteristic length scales under consideration, say $L$, satisfy that $L \gg l_{p}$ [21]. This can be qualitatively argued by estimating the magnitude of the different contributions to the effective action for the gravitational field, considering the relevant Feynman diagrams and using dimensional arguments. Let us write the effective gravitational action, again in a very schematic way, as

$$
S_{\mathrm{eff}}=\int \sqrt{-g}\left(\frac{1}{l_{p}^{2}} R+\alpha R^{2}+l_{p}^{2} R^{3}+\ldots\right),
$$

where $R$ is the Ricci scalar. The first term is the usual classical Einstein-Hilbert term, the second stands for terms quadratic in the curvature (square of Ricci and Weyl tensors) this terms appear as radiative corrections due to vacuum polarization of matter fields, here $\alpha$ is an dimensionless parameter presumably of order 1 , the $R^{3}$ terms are higher order corrections which appear for instance when one considers internal graviton propagators inside matter loops. Let us assume that $R \sim L^{-2}$ then the different terms in the action are of the order of $R^{2} \sim L^{-4}$ and $l_{p}^{2} R^{3} \sim l_{p}^{2} L^{-6}$. Consequently when $L \gg l_{p}^{2}$, the term due to matter loops is a small correction to the Einstein-Hilbert term $\left(1 / l_{p}^{2}\right) R \gg R^{2}$, and this term can be treated as a perturbation. The justification of the order reduction prescription is actually based on this fact. Therefore, significant effects from the vacuum polarization of the matter fields are only expected when their small corrections accumulate in time, as would be the case, for instance, for an evaporating macroscopic black hole all the way before reaching Planckian scales.

However if we have a large number $N$ of matter fields the estimates for the different terms change in a remarkable way. This is interesting because the large $N$ expansion seems the best justification for semiclassical gravity. In fact, now the vacuum polarization terms involving loops of matter are of or$\operatorname{der} N R^{2} \sim N L^{-4}$. For this reason the contribution of the graviton loops, which is of order $R^{2}$, can be neglected in front of the matter loops; this justifies the semiclassical limit. Similarly higher order corrections are of order $N l_{p}^{2} R^{3} \sim N l_{p}^{2} L^{-6}$. Now there is a regime, when $L \sim \sqrt{N} l_{p}$, where the Einstein-Hilbert term is comparable to the vacuum polarization of matter fields, $\left(1 / l_{p}^{2}\right) R \sim N R^{2}$, and yet the higher correction terms can be neglected because we still have $L \gg l_{p}$, provided $N \gg 1$. This is the kind of situation considered in trace anomaly driven inflationary models [55], such as that originally proposed by Starobinsky [58], where the exponential inflation is driven by a large number of massless conformal fields. The order reduction prescription would completely discard the effect from the vacuum polarization of the matter fields even though it is comparable to the Einstein-Hilbert term. In contrast, the procedure proposed by Hawking et al. keeps the contribution from the matter fields. Note that here the actual physical Planck length $l_{p}$ is considered, not the rescaled one, $\vec{l}_{p}^{2}=\overline{\mathrm{\kappa}} / 8 \pi$, which is related to $l_{p}$ by $l_{p}^{2}=\kappa / 8 \pi=\vec{l}_{p}^{2} / N$.

\section{CONCLUSIONS}

An analysis of the stability of any solution of semiclassical gravity with respect to small quantum corrections should consider not only the evolution of the expectation value of the metric perturbations around that solution, but also their fluctuations, encoded in the quantum correlation functions. Making use of the equivalence (to leading order in $1 / N$, where $N$ is the number of matter fields) between the stochastic correlation functions obtained in stochastic semiclassical gravity and the quantum correlation functions for metric perturbations around a solution of semiclassical gravity, the symmetrized two-point quantum correlation function for the metric perturbations can be decomposed into two different parts: the intrinsic fluctuations due to the fluctuations of the initial state of the metric perturbations itself, and the fluctuations induced by their interaction with the matter fields. If one considers the linearized perturbations of the semiclassical Einstein equation, only information on the intrinsic fluctuations can be retrieved. On the other hand, the information on the induced fluctuations naturally follows from the solutions of the Einstein-Langevin equation.

As a specific example, we have analyzed the symmetrized two-point quantum correlation function for the metric perturbations around the Minkowski spacetime interacting with $N$ scalar fields initially in the Minkowski vacuum state. Once the ultraviolet instabilities which are ubiquitous in semiclassical gravity and are commonly regarded as unphysical, have been properly dealt with by using the order reduction prescription or the procedure proposed by Hawking et al. [55, 56], both the intrinsic and the induced contributions to the quantum correlation function for the metric perturbations are found to be stable [26]. Thus, we conclude that Minkowski spacetime is a valid solution of semiclassical gravity.

\section{ACKNOWLEDGMENTS}

I am very grateful to Hans-Thomas Elze and co-organizers for giving me the opportunity to participate at the Second International Workshop DICE2004 on From Decoherence and Emergent Classicality to Emergent Quantum Mechanics, and for their kind and generous hospitality. The research reported here is mainly based in work made in collaboration with BeiLok $\mathrm{Hu}$ and Albert Roura. I am grateful to them for their invaluable contribution to this topic and for having enjoyed many fruitful discussions. I thank Daniel Arteaga, Lajos Diosi, Jonathan Halliwell, Jim Hartle, and Renaud Parentani for discussions on this topic. I also thank Albert Roura for a critical reading of the manuscript and many fruitful discussions. This work has been partially supported by the MEC Research Projects No. FPA2001-3598 and FPA2004-04582. 
[1] N. D. Birrell and P. C. W. Davies, Quantum fields in curved space (Cambridge University Press, Cambridge, 1982).

[2] R. M. Wald, Quantum field theory in curved spacetime and black hole thermodynamics (The University of Chicago Press, Chicago, 1994).

[3] R. M. Wald, Commun. Math. Phys. 54, 1 (1977).

[4] R. M. Wald, Phys. Rev. D 17, 1477 (1978).

[5] R. M. Wald, Ann. Phys. 110, 472 (1978).

[6] L. H. Ford, Ann. Phys. (NY) 144, 238 (1982).

[7] C. I. Kuo and L. H. Ford, Phys. Rev. D 47, 4510 (1993).

[8] B. L. Hu and N. G. Phillips, Int. J. Theor. Phys. 39, 1817 (2000).

[9] N. G. Phillips and B. L. Hu, Phys. Rev. D 62, 084017 (2000).

[10] L. H. Ford and N. F. Svaiter, Phys. Rev. D 56, 226 (1997).

[11] H. Yu and L. H. Ford, Phys. Rev. D 60, 084023 (1999).

[12] H. Yu and L. H. Ford, Phys. Lett. B 496, 107 (2000).

[13] L. H. Ford, Int. J. Theor. Phys. 38, 2941 (1999).

[14] L. H. Ford and C. H. Wu, Int. J. Theor. Phys. 42, 15 (2003).

[15] J. Borgman and L. H. Ford (2003), gr-qc/0307043.

[16] G. T. Horowitz, Phys. Rev. D 21, 1445 (1980).

[17] G. T. Horowitz, in Quantum gravity 2: a second Oxford symposium, edited by C. J. Isham, R. Penrose and D. W. Sciama (Clarendon Press, Oxford, United Kingdom, 1981).

[18] R. D. Jordan, Phys. Rev. D 36, 3593 (1987).

[19] J. Z. Simon, Phys. Rev. D 41, 3720 (1990).

[20] J. Z. Simon, Phys. Rev. D 43, 3308 (1991).

[21] E. E. Flanagan and R. M. Wald, Phys. Rev. D 54, 6233 (1996).

[22] L. Parker and J. Z. Simon, Phys. Rev. D 47, 1339 (1993).

[23] P. R. Anderson, C. Molina-París and E. Mottola, Phys. Rev. D 67, 024026 (2003).

[24] A. Ashtekar, Phys. Rev. Lett. 77, 4864 (1996).

[25] C. Beetle, Adv. Theor. Math. Phys. 2, 471 (1998).

[26] B. L. Hu, A. Roura and E. Verdaguer, Phys. Rev. D 70, 044002 (2004).

[27] B. L. Hu, A. Roura and E. Verdaguer, Int. J. Theor. Phys. 43, 105008 (2004).

[28] B. L. Hu and E. Verdaguer, Class. Quant. Grav. 20, R1 (2003).

[29] B. L. Hu and E. Verdaguer, Living Rev. Rel. 7, 3 (2004).

[30] C. W. Misner, K. S. Thorne and J. A. Wheeler, Gravitation (Freeman, San Francisco, 1973).
[31] J. B. Hartle and G. T. Horowitz, Phys. Rev. D 24, 257 (1981).

[32] W. H. Zurek, Physics Today 44, 36 (1991).

[33] R. Martín and E. Verdaguer, Phys. Rev. D 60, 084008 (1999).

[34] R. Martín and E. Verdaguer, Phys. Rev. D 61, 124024 (2000).

[35] A. Roura and E. Verdaguer, Int. J. Theor. Phys. 39, 1831 (2000).

[36] R. P. Feynman and F. L. Vernon, Ann. Phys. (NY) 24, 118 (1963).

[37] R. P. Feynman and A. R. Hibbs, Quantum mechanics and path integrals (McGraw-Hill, New York, 1965).

[38] J. Schwinger, J. Math. Phys. 2, 407 (1961).

[39] L. V. Keldysh, Zh. Eksp. Teor. Fiz 47, 1515 (1964), [Sov. Phys. JETP 20, 1018 (1965)].

[40] K. Chou, Z. Su, B. Hao and L. Yu, Phys. Rep. 118, 1 (1985).

[41] R. D. Jordan, Phys. Rev. D 33, 444 (1986).

[42] E. Calzetta and B. L. Hu, Phys. Rev. D 35, 495 (1987).

[43] A. Campos and E. Verdaguer, Phys. Rev. D 49, 1861 (1994).

[44] E. Calzetta and B. L. Hu, Phys. Rev. D 49, 6636 (1994).

[45] B. L. Hu and A. Matacz, Phys. Rev. D 51, 1577 (1995).

[46] B. L. Hu and S. Sinha, Phys. Rev. D 51, 1587 (1995).

[47] A. Campos and E. Verdaguer, Phys. Rev. D 53, 1927 (1996).

[48] E. Calzetta, A. Campos and E. Verdaguer, Phys. Rev. D 56, 2163 (1997).

[49] R. Martín and E. Verdaguer, Int. J. Theor. Phys. 38, 3049 (1999).

[50] R. Martín and E. Verdaguer, Phys. Lett. B 465, 113 (1999).

[51] A. Roura and E. Verdaguer, in preparation.

[52] E. Calzetta, A. Roura and E. Verdaguer, Physica A 319, 188 (2003).

[53] E. Calzetta, A. Roura and E. Verdaguer, Phys. Rev. D 64, 105008 (2001).

[54] E. Calzetta, A. Roura and E. Verdaguer, Phys. Rev. Lett. 88, 010403 (2002).

[55] S. W. Hawking, T. Hertog and H. S. Reall, Phys. Rev. D 63, 083504 (2001).

[56] S. W. Hawking and T. Hertog, Phys. Rev. D 65, 103515 (2002).

[57] E. Tomboulis, Phys. Lett. B 70, 361 (1977).

[58] A. A. Starobinsky, Phys. Lett. B 91, 99 (1980). 